\title{
Variance-Constrained Multiobjective Control and Filtering for Nonlinear Stochastic Systems: A Survey
}

\author{
Lifeng Ma, ${ }^{1}$ Zidong Wang, ${ }^{2,3}$ Hongli Dong, ${ }^{4,5}$ and Guoliang Wei ${ }^{6}$ \\ ${ }^{1}$ School of Automation, Nanjing University of Science and Technology, Nanjing 210094, China \\ ${ }^{2}$ School of Information Science and Technology, Donghua University, Shanghai 200051, China \\ ${ }^{3}$ Department of Information Systems and Computing, Brunel University, Uxbridge, Middlesex UB8 3PH, UK \\ ${ }^{4}$ Research Institute of Intelligent Control and Systems, Harbin Institute of Technology, Harbin 150001, China \\ ${ }^{5}$ College of Electrical and Information Engineering, Northeast Petroleum University, Daqing 163318, China \\ ${ }^{6}$ Shanghai Key Lab of Modern Optical System, Department of Control Science and Engineering, \\ University of Shanghai for Science and Technology, Shanghai 200093, China
}

Correspondence should be addressed to Zidong Wang; zidong.wang@brunel.ac.uk

Received 2 October 2013; Accepted 6 October 2013

Academic Editor: Bo Shen

Copyright (C) 2013 Lifeng Ma et al. This is an open access article distributed under the Creative Commons Attribution License, which permits unrestricted use, distribution, and reproduction in any medium, provided the original work is properly cited.

\begin{abstract}
The multiobjective control and filtering problems for nonlinear stochastic systems with variance constraints are surveyed. First, the concepts of nonlinear stochastic systems are recalled along with the introduction of some recent advances. Then, the covariance control theory, which serves as a practical method for multi-objective control design as well as a foundation for linear system theory, is reviewed comprehensively. The multiple design requirements frequently applied in engineering practice for the use of evaluating system performances are introduced, including robustness, reliability, and dissipativity. Several design techniques suitable for the multi-objective variance-constrained control and filtering problems for nonlinear stochastic systems are discussed. In particular, as a special case for the multi-objective design problems, the mixed $H_{2} / H_{\infty}$ control and filtering problems are reviewed in great detail. Subsequently, some latest results on the variance-constrained multi-objective control and filtering problems for the nonlinear stochastic systems are summarized. Finally, conclusions are drawn, and several possible future research directions are pointed out.
\end{abstract}

\section{Introduction}

It is widely recognized that, in almost all engineering applications, nonlinearities are inevitable and could not be eliminated thoroughly. Hence, the nonlinear systems have gained more and more research attention, and lots of results have been published. On the other hand, due to the wide appearance of the stochastic phenomena in almost every aspect of our daily life, stochastic systems which have found successful applications in many branches of science and engineering practice have stirred quite a lot of research interests during the past few decades. Therefore, the control and filtering problems for nonlinear stochastic systems have been studied extensively so as to meet ever-increasing demand toward systems with both nonlinearities and stochasticity.
In many engineering control/filtering problems, the performance requirements are naturally expressed by the upper bounds on the steady state covariance which is usually applied to scale the control/estimation precision, one of the most important performance indices of stochastic design problems. As a result, a large number of control and filtering methodologies have been developed to seek a convenient way to solve the variance-constrained design problems, among which the LQG control and Kalman filtering are two representative minimum variance design algorithms.

On the other hand, in addition to the variance constraints, real-world engineering practice also desires the simultaneous satisfaction of many other frequently seen performance requirements including stability, robustness, reliability, and energy constraints, to name but a few key ones. It gives the 


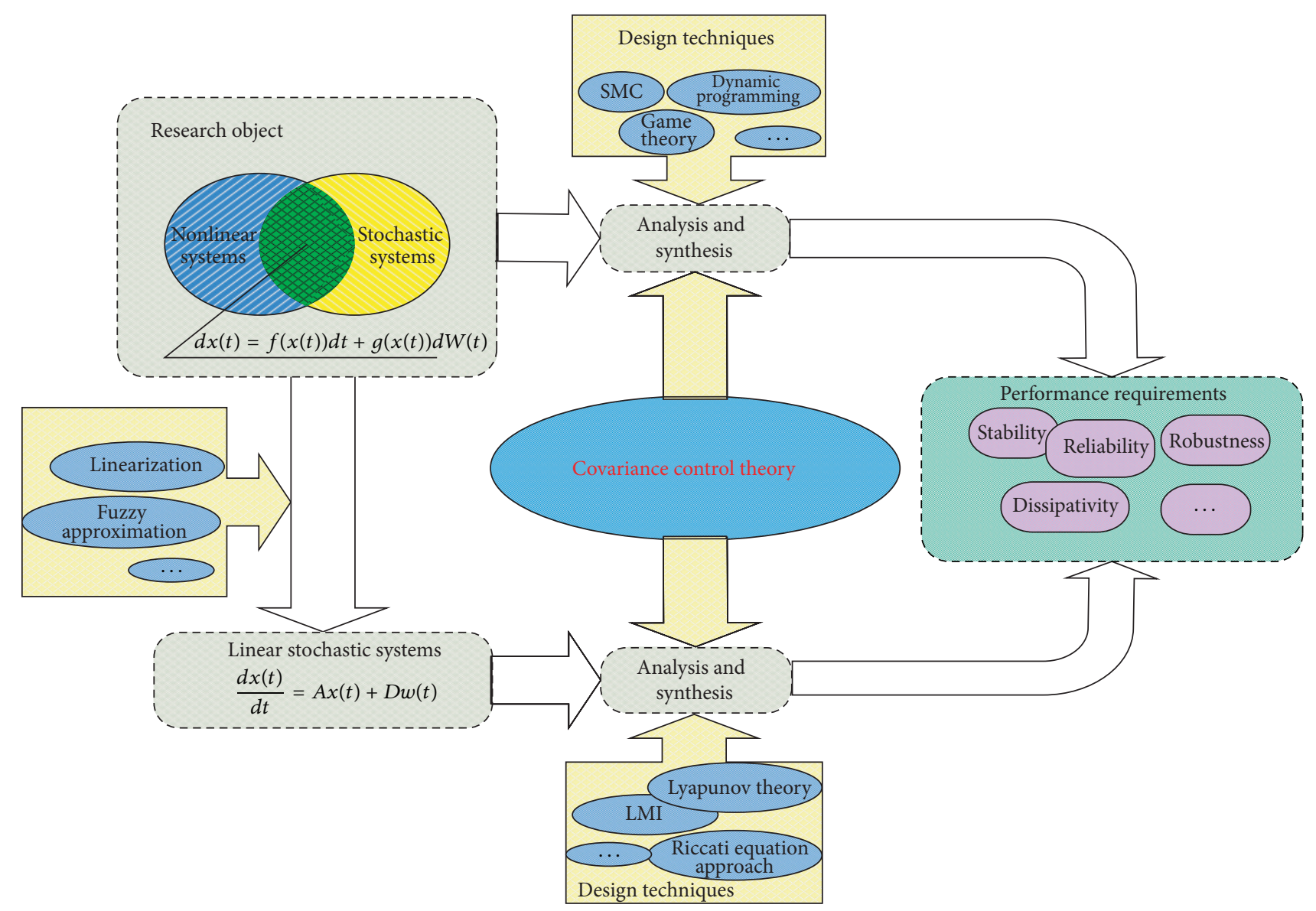

FIGURE 1: Architecture of surveyed contents.

rise to the so-called multiobjective design problems, in which multiple cost functions or performance requirements are simultaneously considered with constraints being imposed on the system. An example of multiobjective control design would be to minimize the system steady-state variance indicating the performance of control precision, subject to a prespecified external disturbance attenuation level evaluating system robustness. Obviously, multiobjective design methods have the ability to provide more flexibility in dealing with the tradeoffs and constraints in a much more explicit manner on the prespecified performance requirements than those conventional optimization methodologies like LQG control scheme or $H_{\infty}$ design technique, which does not seem to have the ability of handling multiple performance specifications.

When coping with the multiobjective design problem with variance constraints for stochastic systems, the wellknown covariance control theory provides us with a useful tool for the system analysis and synthesis. For linear stochastic systems, it has been shown that multiobjective control/filtering problems can be formulated using linear matrix inequalities (LMIs), due to their ability to include desirable performance objectives such as variance constraints, $\mathrm{H}_{2}$ performance, $H_{\infty}$ performance, and pole placement as convex constraints. However, as the nonlinear stochastic systems are concerned, the relevant progress so far has been very slow due primarily to the difficulties in dealing with the variance related problems resulting from the complexity of the nonlinear dynamics. A key issue for the nonlinear covariance control study is the existence of the covariance of nonlinear stochastic systems and its mathematical expression, which is extremely difficult to investigate because of the complex coupling of nonlinearities and stochasticity. Therefore, it is not surprising that the multiobjective control and filtering problems for nonlinear stochastic systems with variance constraints have not been adequately investigated despite their clear engineering insights and good application prospect.

In this paper, we focus mainly on the multiobjective control and filtering problems for nonlinear systems with variance constraints and aim to give a comprehensive survey on some recent advances in this area. The design objects (nonlinear stochastic system), design requirements (multiple performance specifications including variance constraints), several design techniques, and a special case of the addressed problem, mixed $\mathrm{H}_{2} / \mathrm{H}_{\infty}$ design problem, have been discussed in great detail with some recent advances. The contents that are reviewed in this paper and the architecture are shown in Figure 1.

The rest of the paper is organized as follows. In Section 2, the nonlinear stochastic systems are reviewed with some 
recent advances. Section 3 reviews the covariance control theory. Several widely applied performance requirements in engineering practice and commonly seen design techniques in the addressed multiobjective problems are then discussed. Moreover, a special case of multiobjective control and filtering problems, namely, mixed $H_{2} / H_{\infty}$ design problem, is surveyed in great detail. Section 4 gives latest results on multiobjective control and filtering problems of nonlinear stochastic systems with variance constraints. The conclusions and future work are given in Section 5.

\section{Analysis and Synthesis of Nonlinear Stochastic Systems}

For several decades, nonlinear stochastic systems have been attracting increasing attention in the system and control community due to their extensive applications in a variety of areas ranging from communication and transportation to manufacturing, building automation, computing, automotive, and chemical industry, to mention just a few key areas. In this section, the analysis and synthesis problems for nonlinear systems and stochastic systems are recalled, respectively, and some recent advances in these areas are also given.

2.1. Nonlinear Systems. It is well recognized that in almost all engineering applications, nonlinearities are inevitable and could not be eliminated thoroughly. Hence, the nonlinear systems have gained more and more research attention, and lots of results have been reported; see, for example, [1-4]. When analyzing and designing nonlinear dynamical systems, there are a wide range of nonlinear analysis tools, among which the most common and wildly used is linearization because of the powerful tools we know for linear systems. It should be pointed out that, however, there are two basic limitations of linearization [5]. (i) As is well known, linearization is an approximation in the neighborhood of certain operating points. Thus, the resulting linearized system can only show the local behavior of the nonlinear system in the vicinity of those points. Neither nonlocal behavior of the original nonlinear system far away from those operating points nor global behavior throughout the entire state space can be correctly revealed after linearization. (ii) The dynamics of a nonlinear system are much richer than those of a linear system. There are essentially nonlinear phenomena, like finite escape time, multiple isolated equilibria, subharmonic, harmonic, or almost periodic oscillations, to name just a few key ones which can take place only in the presence of nonlinearity; hence, they cannot be described by linear models [69]. Therefore, as a compromise, during the past few decades, there has been tremendous interest in studying nonlinear systems with nonlinearities being taken as the exogenous disturbance input to a linear system, since it could better illustrate the dynamics of the original nonlinear system than the linearized one with less sacrifice of the convenience on the application of existing mathematical tools. The nonlinearities emerging in such systems may arise from the linearization process of an originally highly nonlinear plant or may be an external nonlinear input, which would drastically degrade the system performance or even cause instability; see, for example, [10-13].

On the other hand, in real-world applications, one of the most inevitable and physically important features of some sensors and actuators is that they are always corrupted by different kinds of nonlinearities, either from within the devices themselves or from the external disturbances. Such nonlinearities are generally resulting from equipment limitations as well as the harsh environments such as uncontrollable elements (e.g., variations in flow rates, temperature) and aggressive conditions (e.g., corrosion, erosion, and fouling) [14]. Since the sensor/actuator nonlinearity cannot be simply ignored and often leads to poor performance of the controlled system, a great deal of effort in investigating the analysis and synthesis problems has been devoted by many researchers to the study of various systems with sensor/actuator nonlinearities; see [15-20].

Recently, the system with randomly occurring nonlinearities (RONs) has started to stir quite a lot of research interests as it reveals an appealing fact that, instead of occurring in a deterministic way, a large quantity of nonlinearities in realworld systems would probably take place in a random way. Some of the representative publications can be discussed as follows. The problem of randomly occurring nonlinearities was raised in [21], where an iterative filtering algorithm has been proposed for the stochastic nonlinear system in presence of both RONs and output quantization effects. The filter parameters can be obtained by resorting to solving certain recursive linear matrix inequalities. The obtained results have been soon extended to the case of multiple randomly occurring nonlinearities in [22]. Such a breakthrough on how to deal with nonlinear systems with RONs has been well recognized and quickly followed by other researchers in the area. Using similar techniques, the filtering as well as control problems have been solved for a wide range of systems containing nonlinearities that are occurring randomly, like Markovian jump systems in [23, 24], sliding mode control systems in [25], discrete-time complex networks in [26], sensor networks in [27], time-delay systems in [28], and other types of nonlinear systems [29-31], which therefore has proven that the method developed in [21] is quite general and is applicable to the analysis and synthesis of many different kinds of nonlinear systems.

It should be emphasized that, for nonlinearities, there are many different constraints conditions for certain aim, such as Lipschitz conditions, among which the kind of stochastic nonlinearities described by statistical means has drawn particular research focus since it covers several wellstudied nonlinearities in stochastic systems; see [29, 32-35] and the references therein. Several techniques for analysis and synthesis of this type of nonlinear systems have been exploited, including linear matrix inequality approach [32], Riccati equation method [33], recursive matrix inequality approach [34], gradient method [35], sliding mode control scheme [36], and the game theory approach [29].

2.2. Stochastic Systems. As is well known, in the past few decades, there have been extensive study and application 
of stochastic systems because the stochastic phenomenon is inevitable and cannot be avoided in the real-world systems. When modeling such kinds of systems, the way neglecting the stochastic disturbances, which is a conventional technique in traditional control theory for deterministic systems, is not suitable anymore. Having realized the necessity of introducing more realistic models, nowadays, a great number of realworld systems such as physical systems, financial systems, and ecological systems, as well as social systems, are more suitable to be modeled by stochastic systems, and therefore the stochastic control problem which deals with dynamical systems, described by difference or differential equations, and subject to disturbances characterized as stochastic processes has drawn much research attention; see [37] and the references therein. It is worth mentioning that a kind of stochastic systems represented as deterministic system adding a stochastic disturbance characterized as white noise has gained special research interests and found extensively applications in engineering based on the fact that it is possible to generate stochastic processes with covariance functions belonging to a large class simply by sending white noise through a linear system; hence, a large class of problems can be reduced to the analysis of linear systems with white noise inputs; see [38-42] for examples.

Parallel to the control problems, the filtering and prediction theory for stochastic systems which aims to extract a signal from observations of signals and disturbances has been well studied and found widely applied in many engineering fields. It also plays a very important role in the solution of the stochastic optimal control problem. The research on filtering problem was originated in [43], where the well-known Wiener-Kolmogorov filter has been proposed. However, the Wiener-Kolmogorov filtering theory has not been widely applied mainly because it requires the solution of an integral equation (the Wiener-Hopf equation) which is not easy to solve either analytically or numerically. In [44, 45], Kalman and Bucy gave a significant contribution to the filtering problem, by giving the celebrated Kalman-Bucy filter which could solve the filtering problem recursively. Kalman-Bucy filter (also known as $\mathrm{H}_{2}$ filter) has been extensively adopted and widely used in many branches of stochastic control theory, since the fast development of digital computers recently; see [46-49] and the references therein.

\section{Multiobjective Control and Filtering with Variance Constraints}

In this section, we first review the covariance control theory which provides us with a powerful tool in varianceconstrained design problems with multiple requirements specified by the engineering practice. Then, we discuss several important performance specifications including robustness, reliability, and dissipativity. Two common techniques for solving the addressed problems for nonlinear stochastic systems are introduced. The mixed $H_{2} / H_{\infty}$ design problem is reviewed in great detail as a special case of multiobjective control/filtering problem with variance constraints.
3.1. Covariance Control Theory. As we have stated in the previous section, engineering control problems always require upper bounds on the steady state covariances [41, 50, 51]. However, many control design techniques used in both theoretical analysis and engineering practice, such as LQG and $H_{\infty}$ design, do not seem to give a direct solution to this kind of design problem since they lack a convenient avenue for imposing design objectives stated in terms of upper bounds on the variance values. For example, the LQG controllers minimize a linear quadratic performance index without guaranteeing the variance constraints with respect to individual system states. The covariance control theory [52] developed in the late 80 s has provided a more direct methodology for achieving the individual variance constraints than the LQG control theory. The covariance control theory aims to solve the variance-constrained control problems while satisfying other performance indices $[40,47,52,53]$. It has been shown that the covariance control approach is capable of solving multiobjective design problems, which has found applications in dealing with transient responses, round off errors in digital control, residence time/probability in aiming control problems, and stability, robustness in the presence of parameter perturbations [53]. Such an advantage is based on the fact that several control design objectives, such as stability, time-domain and frequency-domain performance specifications, robustness, and pole location, can be directly related to steady-state covariances of the closed-loop systems. Therefore, covariance control theory serves as a practical method for multiobjective control design as well as a foundation for linear system theory.

On the other hand, it is always the case in real-world applications such as the tracking of a maneuvering target, that the filtering precision is characterized by the error variance of estimation [53, 54]. Considering its clear engineering insights, in the past few years, the filtering problem with error variance constraints has received much interests and a large amount of research fruit has been reported in the literature $[44,45,55,56]$. The celebrated Kalman filtering approach is a typical method which aims to obtain the state information based on the minimization of the variance of the estimation error $[44,45]$. Nevertheless, the strict request of a highly accurate model seriously impedes the application of Kalman filtering as in many cases only an approximate model of the system is available. It therefore has brought about remarkable research interests to the robust filtering method which aims to minimize the error variance of estimation against the system uncertainties or external unknown disturbances [57, 58]. Despite certain merits and successful applications, as in the case of LQG control problem, the traditional minimum variance filtering techniques cannot directly impose the designing objectives stated in terms of upper bounds on the error variance values, by which we mean that those techniques try to minimize the filtering error variance in mean square sense rather than to constrain it within a prespecified bound, which is obviously better to meet the requirements of practical engineering. Motivated by the covariance control theory, in [59], the authors have proposed a more direct designing procedure for achieving the individual variance constraint in filtering problems. Due to 
its design flexibility, the covariance control theory is capable of solving the error variance-constrained filtering problem while guaranteeing other multiple designing objectives [60]. Therefore, it always serves as one of the most powerful tools in dealing with the multiobjective filtering as well as control problems [61].

It should be pointed out that most of the available literature regarding covariance control theory has been concerned with linear time invariant stochastic systems with the linear matrix inequality (LMI) approach. Moreover, when it comes to the variance-constrained controller/filter design problems for much more complicated systems such as time-varying systems, nonlinear systems, and Markovian Jump systems unfortunately, the relevant results have been very few due primarily to the difficulties in dealing with the existence problem of the steady-state covariances and their mathematical expressions for the abovementioned complex systems. With the hope to resolve such difficulties, in recent years, special efforts have been devoted in study of the varianceconstrained multiobjective design problems for systems of complex dynamics, and several methodologies for analysis and synthesis have been developed. For example, in [47], a Riccati equation method has been proposed to solve the filtering problem for linear time-varying stochastic systems with prespecified error variance bounds. In [62-64], by means of the technique of sliding mode control (SMC), robust controller design problem has been solved for linear parameter perturbed systems, since SMC has certain robustness to matched disturbances or parameter perturbations. We shall return to this SMC problem later, and more details will be discussed in the following section.

When it comes to nonlinear stochastic systems, limited work has been done in the covariance-constrained analysis and design problems, just as what we have anticipated. A multiobjective filter has been designed in [65] for systems with Lipschitz-type nonlinearity, but the variance bounds cannot be prespecified. Strictly speaking, such an algorithm cannot be referred to as variance-constrained filtering in view of lack of capability for directly imposing specified constraints on variance. An LMI approach has been proposed in [32] to cope with robust filtering problems for a class of stochastic systems with nonlinearities characterized by statistical means, attaining an assignable $H_{2}$ performance index. In [61], for a special class of nonlinear stochastic systems, namely, systems with multiplicative noises (also called bilinear systems or systems with state/control dependent noises), a state feedback controller has been put forward in a unified LMI framework in order to ensure that the multiple objectives including stability, $H_{\infty}$ specification, and variance constraints are simultaneously satisfied. This paper is always regarded as the origination of covariance control theory for nonlinear systems, as within the established theoretical framework, quite a lot of performance requirements can be taken into consideration simultaneously. Furthermore, with the developed techniques, the obtained elegant results could be easily extended to a wide range of nonlinear stochastic systems; see, for example, [29, 35, 66-68]. We shall return to such a type of nonlinear stochastic systems later to present more details of recent progresses in Section 4.
3.2. Multiple Performance Requirements. In the following, several performance indices originated from the engineering practice and frequently applied in multiobjective design problems are introduced.

3.2.1. Robustness. In real-world engineering practice, various reasons such as variations of the operating point, aging of devices, and identification errors, would lead to the parameter uncertainties which result in the perturbations of the elements of a system matrix when modeling the system in a state-space form. Such a perturbation in system parameters cannot be avoided and would cause degradation (sometimes even instability) to the system performance. Therefore, in the past decade, considerable attention has been devoted to different issues for linear or nonlinear uncertain systems, and a great number of papers have been published; see $[2,48,69-74]$ for some recent results.

On another research frontier of robust control, the $H_{\infty}$ design method which is used to design controller/filter with guaranteed performances with respect to the external disturbances as well as internal perturbations has received an appealing research interest during the past decades; see [75-78], for instance. Since Zames' original work [75], significant advances have been made in the research area of $H_{\infty}$ control and filtering. The standard $H_{\infty}$ control problem has been completely solved by Doyle et al. for linear systems by deriving simple state-space formulas for all controllers [76]. For nonlinear systems, the $H_{\infty}$ performance evaluation can be conducted through analyzing the $L_{2}$ gain of the relationship between the external disturbance and the system output, which is a necessary step to decide whether further controller design is needed. In the past years, the nonlinear $H_{\infty}$ control problem has also received considerable research attention, and many results have been available in the literature [77-81]. On the other hand, the $H_{\infty}$ filtering problem has also gained considerable research interests along with the development of $H_{\infty}$ control theory; see [26, 79, 82-85]. It is well known that the existence of a solution to the $H_{\infty}$ filtering problem is in fact associated with the solvability of an appropriate algebraic Riccati equality (for the linear cases) or a so-called Hamilton-Jacobi equation (for the nonlinear ones). So far, there have been several approaches for providing solutions to nonlinear $H_{\infty}$ filtering problems, few of which, however, are capable of handling multiple performance requirements in an $H_{\infty}$ optimization framework.

It is worth mentioning that, in contrast to the $H_{\infty}$ design framework within which multiple requirements can hardly be under simultaneous consideration, the covariance control theory has provided a convenient avenue for the robustness specifications to be perfectly integrated into the multiobjective design procedure; see $[61,80]$, for example. For nonlinear stochastic systems, control and filtering problems have been solved with the occurrence of parameter uncertainties and stochastic nonlinearities while guaranteeing the $H_{\infty}$ and variance specifications; see $[35,66,67,80]$ for some recent publications. 
3.2.2. Reliability. In practical control systems especially networked control systems (NCSs), due to a variety of reasons including the erosion caused by severe circumstance, abrupt changes of working conditions, the intense external disturbance, and the internal physical equipment constraints and aging, the process of signal sampling and transmission has always confronted with different kinds of failures such as measurements missing, signal quantization, and sensor and actuator saturations. Such a phenomenon is always referred to as incomplete information, which would drastically degrade the system performance. In recent years, as requirements increase toward the reliability of engineering systems, the reliable control problem which aims to stabilize the systems accurately and precisely in spite of incomplete information caused by possible failures has therefore attracted considerable attention. In $[86,87]$, binary switching sequences and Markovian jumping parameters have been introduced to model the measurements missing phenomena. A more general model called the multiple measurements missing model has been proposed in [88] by employing a diagonal matrix to characterize the different missing probabilities for individual sensors. The incomplete information caused by sensor and actuator saturations is also receiving considerable research attention, and some results have been reported in the literature $[20,89,90]$, where the saturation has been modeled as so-called sector bound nonlinearities. As far as signal quantization is mentioned, in [19], a sector bound scheme has been proposed to handle the logarithmic quantization effects in feedback control systems, and such an elegant scheme has then been extensively employed later on; see, for example, $[91,92]$ and the references therein.

It should be pointed out that, for nonlinear stochastic systems, the relevant results of reliable control/filtering with variance constraints are relatively fewer, and some representative results can be summarized as follows. By means of linear matrix inequality approach, a reliable controller has been designed for nonlinear stochastic system in [66] against actuator faults with variance constraints. In the case of sensor failures, the gradient method and LMI method have been applied, respectively, in [35] and [67] to design multiobjective filters, respectively, satisfying multiple requirements including variance specifications simultaneously. However, despite its clear physical insight and importance in engineering application, the control problem for nonlinear stochastic systems with incomplete information has not yet been studied sufficiently.

3.2.3. Dissipativity. In recent years, the theory of dissipative systems, which plays an important role in system and control areas, has been attracting a great deal of research interests, and many results have been reported so far; see [93-99]. Originated in [97], the dissipative theory serves as a powerful tool in characterizing important system behaviors such as stability and passivity and has close connections with bounded real lemma, passivity lemma, and circle criterion. It is worth mentioning that, due to its simplicity in analysis and convenience in simulation, the LMI method has gained particular attention in dissipative control problems. For example, in $[96,98]$, an LMI method was used to design the state feedback controller ensuring both the asymptotic stability and strictly quadratic dissipativity. For singular systems, [93] has established a unified LMI framework to satisfy admissibility and dissipativity of the system simultaneously. In [95], the dissipative control problem has been solved for time-delay systems.

Although the dissipativity theory provides us a useful tool for the analysis of systems with multiple performance criteria, unfortunately, when it comes to nonlinear stochastic systems, few of the literature has been concerned with the multiobjective design problem for nonlinear stochastic systems, except [100], where a multiobjective control law has been proposed to simultaneously meet the stability, variance constraints, and dissipativity of closed-loop system. So far, the variance-constrained design problem with dissipativity being taken into consideration has not yet been studied adequately and is still remaining challenging.

\subsection{Design Techniques for Nonlinear Stochastic Systems with} Variance Constraints. The complexity of nonlinear system dynamics challenges us to come up with systematic design procedures to meet control objectives and design specifications. It is clear that we cannot expect one particular procedure to apply to all nonlinear systems; therefore, quite a lot of tools have been developed to deal with control and filtering problems for nonlinear stochastic systems, including T-S fuzzy model approximation approach, linearization, gain scheduling, sliding mode control, and backstepping, to name but a few key ones. In the sequel, we will investigate two nonlinear design tools that can be well combined with the covariance control theory for the purpose of providing a theoretical framework within which the variance-constrained control and filtering problems can be solved systematically for nonlinear stochastic systems.

3.3.1. T-S Fuzzy Model. The T-S fuzzy model approach occupies an important place in the study of nonlinear systems for its excellent capability in nonlinear system descriptions. Such a model allows one to perfectly approximate a nonlinear system by a set of local linear subsystems with certain fuzzy rules, thereby carrying out the analysis and synthesis work within the linear system framework. Therefore, T-S fuzzy model is extensively applied in both theoretical research and engineering practice of nonlinear systems; see [101-104] for some latest publications. However, despite its engineering significance, few of the literature has taken the system state variance into consideration mainly due to the technical difficulties in dealing with the variance related problems. Some tentative work can be summarized as follows. In [105], a minimum variance control algorithm as well as direct adaptive control scheme has been applied in a stochastic T$S$ fuzzy ARMAX model to track the desired reference signal. However, as we mentioned above, such a minimum variance control algorithm lacks the ability of directly imposing design requirements on the variances of individual state component. Therefore, in order to cope with this problem, in [106], 
a fuzzy controller has been designed to stabilize a nonlinear continuous-time system, while simultaneously minimizing the control input energy and satisfying variance constraints placed on the system state. The result has then been extended in [107] to the output variance constraints case. Recently, such a T-S fuzzy model based varianceconstrained algorithm has found successful application in nonlinear synchronous generator systems; see [108] for more details.

3.3.2. Sliding Mode Control. In the past few decades, the sliding mode control (also known as variable structure control) problem originated in [109] has been extensively studied and widely applied, because of its advantage of strong robustness against model uncertainties, parameter variations, and external disturbances. In the sliding mode control, trajectories are forced to reach a sliding manifold in finite time and to stay on the manifold for all future time. It is worth mentioning that in the existing literature about sliding mode control problem for nonlinear systems, the nonlinearities and uncertainties taken into consideration are mainly under the matching conditions, that is, when nonlinear and uncertain terms enter the state equation at the same point as the control input and motion on the sliding manifold is independent of those matched terms; see [110, 111] for examples. Under such an assumption, the covarianceconstrained control problems have been solved in [62-64] for a type of continuous stochastic systems with matching condition nonlinearities.

Along with the development of continuous-time sliding mode control theory, in recent years, as most control strategies are implemented in a discrete-time setting (e.g., networked control systems), the sliding mode control problem for discrete-time systems has gained considerable research interests, and a large amount of literature has appeared on this topic. For example, in [112, 113], the integral type SMC schemes have been proposed for sample-data systems and a class of nonlinear discrete-time systems, respectively. Adaptive laws were applied in $[114,115]$ to synthesize sliding mode controllers for discrete-time systems with stochastic as well as deterministic disturbances. In [116], a simple methodology for designing sliding mode controllers was proposed for a class of linear multi-input discrete-time systems with matching perturbations. Using dead-beat control technique, [117] presented a discrete variable structure control method with a finite-time step to reach the switching surface. In cases when the system states were not available, the discretetime SMC problems were solved in $[118,119]$ via output feedback. It is worth mentioning that in [120], the discretetime sliding mode reaching condition was first revised, and then a reaching law approach was developed which has proven to be a convenient way to handle robust control problems; see $[121,122]$ for some latest publications. Recently, for discrete-time systems that are not only confronted with nonlinearities but also corrupted by more complicated situations like propagation time delays, randomly occurring parameter uncertainties, and multiple data packet dropouts, the SMC strategies have been designed in $[25,81,83]$ to solve the robust control problems and have shown good performances against all the mentioned negative factors. Currently, the sliding mode control problems for discretetime systems still remain a hotspot in systems and control science; however, when it comes to the variance-constrained problems, the related work is much fewer. As preliminary work, [36] has proposed an SMC algorithm guaranteeing the required $\mathrm{H}_{2}$ specification for discrete-time stochastic systems in presence of both matched and unmatched nonlinearities. In this paper, although only the $\mathrm{H}_{2}$ performance is handled, it is worth mentioning that, with the proposed method, other performance indices can be considered simultaneously within the established unified framework by employing similar design techniques.

3.4. A Special Case of Multiobjective Design: Mixed $\mathrm{H}_{2} / \mathrm{H}_{\infty}$ Control/Filtering. As a special case of multiobjective control problem, the mixed $H_{2} / H_{\infty}$ control/filtering has gained a great deal of research interests for several decades. So far, there have been several approaches to tackling the mixed $\mathrm{H}_{2} / \mathrm{H}_{\infty}$ control/filtering problem. For linear deterministic systems, the mixed $H_{2} / H_{\infty}$ control problems have been extensively studied. For example, an algebraic approach has been presented in [123] and a time domain Nash game approach has been proposed in $[39,124]$ to solve the addressed mixed $\mathrm{H}_{2} / \mathrm{H}_{\infty}$ control/filtering problems, respectively. Moreover, some efficient numerical methods for mixed $\mathrm{H}_{2} / \mathrm{H}_{\mathrm{\infty}}$ control problems have been developed based on a convex optimization approach in $[42,125-127]$, among which the linear matrix inequality approach has been employed widely to design both linear state feedback and output feedback controllers subject to $\mathrm{H}_{2} / \mathrm{H}_{\mathrm{\infty}}$ criterion due to its effectiveness in numerical optimization. It is noted that the mixed $H_{2} / H_{\infty}$ control theories have already been applied to various engineering fields $[49,128,129]$.

Parallel to the mixed $\mathrm{H}_{2} / \mathrm{H}_{\infty}$ control problem, the mixed $\mathrm{H}_{2} / \mathrm{H}_{\infty}$ filtering problem has also been well studied, and several approaches have been proposed to tackling the problem. For example, Bernstein and Haddad [123] transformed the mixed $\mathrm{H}_{2} / \mathrm{H}_{\infty}$ filtering problem into an auxiliary minimization problem. Then, by using the Lagrange multiplier technique, they gave the solutions in terms of an upper bound on the $\mathrm{H}_{2}$ filtering error. In [130, 131], a time domain game approach was proposed to solve the mixed $\mathrm{H}_{2} / \mathrm{H}_{\infty}$ filtering problem through a set of coupled Riccati equations. Recently, LMI method has been widely employed to solve the multiobjective mixed $\mathrm{H}_{2}$ and $\mathrm{H}_{\mathrm{\infty}}$ filtering problems; see $[60,132]$ for examples.

As far as nonlinear systems are concerned, the mixed $\mathrm{H}_{2} / \mathrm{H}_{\infty}$ control problem as well as filtering problem has gained some research interests; see, for examples, [133135]. For nonlinear deterministic systems, the mixed $\mathrm{H}_{2} / \mathrm{H}_{\infty}$ control problem has been solved with the solutions characterized in terms of the cross-coupled Hamilton-JacobiIsaacs (HJI) partial differential equations. Since it is difficult to solve the cross-coupled HJI partial differential equations either analytically or numerically, in [134], the authors have used the Takagi and Sugeno (T-S) fuzzy linear model to 
approximate the nonlinear system, and solutions to the mixed $\mathrm{H}_{2} / \mathrm{H}_{\infty}$ fuzzy output feedback control problem have been obtained via an LMI approach. For nonlinear stochastic systems, unfortunately, the mixed $H_{2} / H_{\infty}$ control and filtering problem has not received full investigation, and few results have been reported. In [38], for a special type of nonlinear stochastic system, which is known as bilinear systems (also called systems with state-dependent noise or systems with multiplicative noise), a stochastic mixed $\mathrm{H}_{2} / \mathrm{H}_{\infty}$ control problem has been solved and sufficient conditions have been provided in terms of the existence of the solutions of crosscoupled Riccati equations. Very recently, an LMI approach has been proposed in [135] to solve the mixed $H_{2} / H_{\infty}$ control problem for a class of nonlinear stochastic systems which includes several well-studied types of nonlinear systems. For the stochastic systems with much more complicated nonlinearities, by means of game theory approach, the mixed $\mathrm{H}_{2} / \mathrm{H}_{\infty}$ control problem has been solved for systems with RONs in [29] and Markovian jump parameters in [68], respectively. Nevertheless, to the best of authors' knowledge, the mixed $\mathrm{H}_{2} / \mathrm{H}_{\mathrm{\infty}}$ control and filtering problems for general nonlinear systems have not yet received enough investigation and still remain as challenging topics.

\section{Latest Progress}

Very recently, the variance-constrained multiobjective control as well as filtering problem for nonlinear stochastic systems has been intensively studied, and some elegant results have been reported. In this section, we highlight some of the newest work with respect to this topic.

(i) In [67], a robust variance-constrained filter has been designed for a class of nonlinear stochastic systems with both parameter uncertainties and probabilistic missing measurements. In this paper, we have simultaneously considered the exponentially mean-square stability, variance constraints, robustness against the parameter uncertainties, and reliability in case of possible measurements missing. A general framework for solving this problem has been established using an LMI approach.

(ii) For the stochastic system with nonlinearities of both the matched and unmatched forms, in [81], a sliding mode control algorithm has been proposed to solve the robust $\mathrm{H}_{2}$ control problem. A new discrete-time switching function has been proposed, and then a sufficient condition has been derived to ensure both the exponentially mean-square stability and the $\mathrm{H}_{2}$ performance in the sliding surface. It is worth mentioning that, using the proposed method in this paper, several typical classes of stochastic nonlinearities can be dealt with via SMC method.

(iii) In [100], a dissipative control problem has been solved for a class of nonlinear stochastic systems while guaranteeing tumultuously exponentially mean-square stability, variance constraints, system dissipativity, and reliability. An algorithm has been proposed to convert the original nonconvex feasibility problem into an optimal minimization problem which is much more easy to solve by standard numerical software.

(iv) For the same type of nonlinear stochastic systems as mentioned above, in [66], a robust varianceconstrained controller has been designed with the guaranteed reliability against the possible actuator failures.

(v) When the nonlinear stochastic system is timevarying, [80] has designed a multiobjective controller that meets the $H_{\infty}$ performance and variance constraint over a finite horizon. By using the recursive linear matrix inequalities method, a sufficient condition for the solvability of the addressed controller design problem has been given. Such an algorithm is so elegant that it is soon followed by many researchers in related fields.

(vi) When it comes to the finite-horizon multiobjective filtering for time-varying nonlinear stochastic systems, [35] has proposed a technique that could handle $H_{\infty}$ performance and variance constraint at the same time. It is worth mentioning that the design algorithm developed in this paper is forward in time, which is different from those in most of the existing literature where the $H_{\infty}$ problem can be solved only backward in time and thus can be combined with the variance design and is suitable for online design.

(vii) In [29], the mixed $H_{2} / H_{\infty}$ controller design problem has been dealt with for a class of nonlinear stochastic systems with randomly occurring nonlinearities that are characterized by two Bernoulli distributed white sequences with known probabilities. For the multiobjective controller design problem, the sufficient condition of the solvability of the mixed $\mathrm{H}_{2} / \mathrm{H}_{\infty}$ control problem has been established by means of the solvability of four coupled matrix-valued equations. A recursive algorithm has been developed to obtain the value of feedback controller step by step at every sampling instant. Such a design algorithm has been extended to the Markovian Jump systems with probabilistic sensor failures in [68].

\section{Conclusions and Future Work}

In this paper, the variance-constrained multiobjective control and filtering problems have been reviewed with some recent advances for nonlinear stochastic systems. Latest results on analysis and synthesis problems for nonlinear stochastic systems with multiple performance constraints have been surveyed. Based on the literature review, some related topics for the future research work are listed as follows.

In practical engineering, there are still some more complicated yet important kinds of nonlinearities that have not been studied. Therefore, the variance-constrained multiobjective control and filtering problems for more general nonlinear systems still remain open and challenging. 
Another future research direction is to further investigate new performance indices (e.g., system energy constraints) that can be simultaneously considered with other existing ones. Also, variance-constrained multiobjective modeling, estimation, filtering, and control problems could be considered for more complex systems $[4,13,73,74,99]$.

It would be interesting to study the problems of varianceconstrained multiobjective analysis and design for large scale nonlinear interconnected systems that are frequently seen in modern industries.

A practical engineering application of the existing theories and methodologies would be the target tracking problem.

\section{Acknowledgments}

This work was supported in part by the National Natural Science Foundation of China under Grants 61304010, 61134009, 61329301,61374127 , and 61374039, the Royal Society of the UK, and the Alexander von Humboldt Foundation of Germany.

\section{References}

[1] Z. Wang, Y. Liu, and X. Liu, " $H_{\infty}$ filtering for uncertain stochastic time-delay systems with sector-bounded nonlinearities," Automatica, vol. 44, no. 5, pp. 1268-1277, 2008.

[2] Y. Liu, Z. Wang, and X. Liu, "Robust $H_{\infty}$ control for a class of nonlinear stochastic systems with mixed time delay," International Journal of Robust and Nonlinear Control, vol. 17, no. 16, pp. 1525-1551, 2007.

[3] D. Yue and Q.-L. Han, "Delay-dependent exponential stability of stochastic systems with time-varying delay, nonlinearity, and Markovian switching," IEEE Transactions on Automatic Control, vol. 50, no. 2, pp. 217-222, 2005.

[4] Y. Chen and K. A. Hoo, "Stability analysis for closed-loop management of a reservoir based on identification of reduced-order nonlinear model," Systems Science and Control Engineering: An Open Access Journal, vol. 1, no. 1, pp. 12-19, 2013.

[5] H. K. Khalil, Nonlinear Systems, Publishing House of Electronics Industry, Beijing, China, 3rd edition, 2007.

[6] J. Guckenheimer and P. Holmes, Nonlinear Oscillations, Dynamical Systems, and Bifurcations of Vector Fields, vol. 42, Springer, New York, NY, USA, 1983.

[7] J. K. Hale and H. Koçak, Dynamics and Bifurcations, vol. 3, Springer, New York, NY, USA, 1991.

[8] S. Strogatz, Nonlinear Dynamics and Chaos, Addison Wesley, Reading, Mass, USA, 1994.

[9] J. Wait, L. Huelsman, and G. Korn, Introduction to Operational Amplifiers, McGraw-Hill, New York, NY, USA, 1975.

[10] L. Guo and H. Wang, "Fault detection and diagnosis for general stochastic systems using B-spline expansions and nonlinear filters," IEEE Transactions on Circuits and Systems I, vol. 52, no. 8, pp. 1644-1652, 2005.

[11] Z. Wang, Y. Liu, M. Li, and X. Liu, "Stability analysis for stochastic Cohen-Grossberg neural networks with mixed time delays," IEEE Transactions on Neural Networks, vol. 17, no. 3, pp. 814-820, 2006.

[12] Y. Wang, L. Xie, and C. E. de Souza, "Robust control of a class of uncertain nonlinear systems," Systems \& Control Letters, vol. 19, no. 2, pp. 139-149, 1992.
[13] S. R. Desai and R. Prasad, "A new approach to order reduction using stability equation and big bang big crunch optimization," Systems Science and Control Engineering: An Open Access Journal, vol. 1, no. 1, pp. 20-27, 2013.

[14] R. K. Pearson, "Gray-box modeling of nonideal sensors," in Proceedings of the American Control Conference, pp. 4404-4409, Arlington, Va, USA, June 2001.

[15] Y.-Y. Cao, Z. Lin, and B. M. Chen, "An output feedback $H_{\infty}$ controller design for linear systems subject to sensor nonlinearities," IEEE Transactions on Circuits and Systems I, vol. 50, no. 7, pp. 914-921, 2003.

[16] Q.-L. Han, "Absolute stability of time-delay systems with sectorbounded nonlinearity," Automatica, vol. 41, no. 12, pp. 21712176, 2005.

[17] G. Kreisselmeier, "Stabilization of linear systems in the presence of output measurement saturation," Systems \& Control Letters, vol. 29, no. 1, pp. 27-30, 1996.

[18] Z. Lin and T. Hu, "Semi-global stabilization of linear systems subject to output saturation," Systems \& Control Letters, vol. 43, no. 3, pp. 211-217, 2001.

[19] M. Fu and L. Xie, "The sector bound approach to quantized feedback control," IEEE Transactions on Automatic Control, vol. 50, no. 11, pp. 1698-1711, 2005.

[20] Z. Wang, D. W. C. Ho, H. Dong, and H. Gao, "Robust $H_{\infty}$ finite-horizon control for a class of stochastic nonlinear timevarying systems subject to sensor and actuator saturations," IEEE Transactions on Automatic Control, vol. 55, no. 7, pp. 1716$1722,2010$.

[21] B. Shen, Z. Wang, H. Shu, and G. Wei, "Robust $H_{\infty}$ finitehorizon filtering with randomly occurred nonlinearities and quantization effects," Automatica, vol. 46, no. 11, pp. 1743-1751, 2010 .

[22] B. Shen, Z. Wang, H. Shu, and G. Wei, " $H_{\infty}$ filtering for uncertain time-varying systems with multiple randomly occurred nonlinearities and successive packet dropouts," International Journal of Robust and Nonlinear Control, vol. 21, no. 14, pp. 16931709, 2011.

[23] H. Dong, Z. Wang, D. W. C. Ho, and H. Gao, "Robust $H_{\infty}$ filtering for Markovian jump systems with randomly occurring nonlinearities and sensor saturation: the finite-horizon case," IEEE Transactions on Signal Processing, vol. 59, no. 7, pp. 30483057, 2011.

[24] H. Dong, Z. Wang, and H. Gao, "Fault detection for Markovian jump systems with sensor saturations and randomly varying nonlinearities," IEEE Transactions on Circuits and Systems I, vol. 59, no. 10, pp. 2354-2362, 2012.

[25] J. Hu, Z. Wang, H. Gao, and L. K. Stergioulas, "Robust sliding mode control for discrete stochastic systems with mixed time delays, randomly occurring uncertainties, and randomly occurring nonlinearities," IEEE Transactions on Industrial Electronics, vol. 59, no. 7, pp. 3008-3015, 2012.

[26] D. Ding, Z. Wang, J. Hu, and H. Shu, " $H_{\infty}$ state estimation for discrete-time complex networks with randomly occurring sensor saturations and randomly varying sensor delays," IEEE Transactions on Neural Networks and Learning Systems, vol. 23, no. 5, pp. 725-736, 2012.

[27] J. Liang, Z. Wang, B. Shen, and X. Liu, "Distributed state estimation in sensor networks with randomly occurring nonlinearities subject to time-delays," ACM Transactions on Sensor Networks, vol. 9, no. 1, article 4, 2012.

[28] G. Wei, Z. Wang, and B. Shen, "Probability-dependent gainscheduled control for discrete stochastic delayed systems with 
randomly occurring nonlinearities," International Journal of Robust and Nonlinear Control, vol. 23, no. 7, pp. 815-826, 2013.

[29] L. Ma, Z. Wang, Y. Bo, and Z. Guo, "A game theory approach to mixed $\mathrm{H}_{2} / \mathrm{H}_{\infty}$ control for a class of stochastic time-varying systems with randomly occurring nonlinearities," Systems \& Control Letters, vol. 60, no. 12, pp. 1009-1015, 2011.

[30] Z. Wang, B. Shen, and X. Liu, " $H_{\infty}$ filtering with randomly occurring sensor saturations and missing measurements," Automatica, vol. 48, no. 3, pp. 556-562, 2012.

[31] D. Ding, Z. Wang, J. Hu, and H. Shu, "Dissipative control for state-saturated discrete time-varying systems with randomly occurring nonlinearities and missing measurements," International Journal of Control, vol. 86, no. 4, pp. 674-688, 2013.

[32] F. Yang, Z. Wang, D. W. C. Ho, and X. Liu, "Robust $\mathrm{H}_{2}$ filtering for a class of systems with stochastic nonlinearities," IEEE Transactions on Circuits and Systems II, vol. 53, no. 3, pp. 235239, 2006.

[33] E. E. Yaz and Y. I. Yaz, "State estimation of uncertain nonlinear stochastic systems with general criteria," Applied Mathematics Letters, vol. 14, no. 5, pp. 605-610, 2001.

[34] H. Dong, Z. Wang, D. W. C. Ho, and H. Gao, "Varianceconstrained $H_{\infty}$ filtering for a class of nonlinear time-varying systems with multiple missing measurements: the finitehorizon case," IEEE Transactions on Signal Processing, vol. 58, no. 5, pp. 2534-2543, 2010.

[35] L. Ma, Y. Bo, Y. Zhou, and Z. Guo, "Error variance-constrained $H_{\infty}$ filtering for a class of nonlinear stochastic systems with degraded measurements: the finite horizon case," International Journal of Systems Science, vol. 43, no. 12, pp. 2361-2372, 2012.

[36] L. F. Ma, Z. D. Wang, and Z. Guo, "Robust $\mathrm{H}_{2}$ sliding mode control for non-linear discrete-time stochastic systems," IET Control Theory \& Applications, vol. 3, no. 11, pp. 1537-1546, 2009.

[37] K. J. Åström, Introduction to Stochastic Control Theory, Academic Press, New York, NY, USA, 1970.

[38] B.-S. Chen and W. Zhang, "Stochastic $H_{2} / H_{\infty}$ control with state-dependent noise," IEEE Transactions on Automatic Control, vol. 49, no. 1, pp. 45-57, 2004.

[39] X. Chen and K. Zhou, "Multi-objective $H_{2}$ and $H_{\infty}$ control design," SIAM Journal on Control and Optimization, vol. 40, no. 2, pp. 628-660, 2002.

[40] E. G. Collins, Jr. and R. E. Skelton, "A theory of state covariance assignment for discrete systems," IEEE Transactions on Automatic Control, vol. 32, no. 1, pp. 35-41, 1987.

[41] B. Huang, "Minimum variance control and performance assessment of time-variant processes," Journal of Process Control, vol. 12, no. 6, pp. 707-719, 2002.

[42] C. W. Scherer, "Multi-objective $\mathrm{H}_{2} / \mathrm{H}_{\infty}$ control," IEEE Transactions on Automatic Control, vol. 40, no. 6, pp. 1054-1062, 1995.

[43] N. Wiener, The Extrapolation, Interpolation and Smoothing of Stationary Time Series with Engineering Applications, John Wiley \& Sons, New York, NY, USA, 1949.

[44] R. Kalman and R. Bucy, "A new approach to linear filtering and prediction problems," ASME Journal of Basic Engineering, vol. 82, pp. 34-45, 1960.

[45] R. E. Kalman and R. S. Bucy, "New results in linear filtering and prediction theory," ASME Journal of Basic Engineering, vol. 83, pp. 95-108, 1961.

[46] D. S. Bernstein and W. M. Haddad, "Steady-state Kalman filtering with an $H_{\infty}$ error bound," Systems \& Control Letters, vol. 12, no. 1, pp. 9-16, 1989.
[47] Y. S. Hung and F. Yang, "Robust $H_{\infty}$ filtering with error variance constraints for discrete time-varying systems with uncertainty," Automatica, vol. 39, no. 7, pp. 1185-1194, 2003.

[48] X. Lu, L. Xie, H. Zhang, and W. Wang, "Robust Kalman filtering for discrete-time systems with measurement delay," IEEE Transactions on Circuits and Systems II, vol. 54, no. 6, pp. 522-526, 2007.

[49] H. Rotstein, M. Sznaier, and M. Idan, " $H_{2} / H_{\infty}$ filtering theory and an aerospace application," International Journal of Robust and Nonlinear Control, vol. 6, no. 4, pp. 347-366, 1996.

[50] K. Boukas, "Stabilization of stochastic nonlinear hybrid systems," International Journal of Innovative Computing, Information and Control, vol. 1, no. 1, pp. 131-141, 2005.

[51] B. Huang, S. L. Shah, and E. K. Kwok, "Good, bad or optimal? Performance assessment of multivariable processes," Automatica, vol. 33, no. 6, pp. 1175-1183, 1997.

[52] A. Hotz and R. E. Skelton, "Covariance control theory," International Journal of Control, vol. 46, no. 1, pp. 13-32, 1987.

[53] R. Skelton, T. Iwasaki, and K. Grigoriadis, A Unified Approach to Linear Control Design, Taylor \& Francis, London, UK, 1997.

[54] H. Ruan, E. Yaz, and T. Zhai, "Current output filter for state estimation of nonlinear stochastic systems with linear measurement," International Journal of Innovative Computing, Information and Control, vol. 1, no. 2, pp. 277-287, 2005.

[55] U. Shaked and C. E. de Souza, "Robust minimum variance filtering," IEEE Transactions on Signal Processing, vol. 43, no. 11, pp. 2474-2483, 1995.

[56] Z. Wang, D. W. C. Ho, and X. Liu, "Variance-constrained filtering for uncertain stochastic systems with missing measurements," IEEE Transactions on Automatic Control, vol. 48, no. 7, pp. 1254-1258, 2003.

[57] C.-S. Hsieh, "Extension of unbiased minimum-variance input and state estimation for systems with unknown inputs," Automatica, vol. 45, no. 9, pp. 2149-2153, 2009.

[58] U. Shaked, L. Xie, and Y. C. Soh, "New approaches to robust minimum variance filter design," IEEE Transactions on Signal Processing, vol. 49, no. 11, pp. 2620-2629, 2001.

[59] Z. Wang, D. W. C. Ho, and X. Liu, "Robust filtering under randomly varying sensor delay with variance constraints," IEEE Transactions on Circuits and Systems II, vol. 51, no. 6, pp. 320326, 2004.

[60] F. Yang, Z. Wang, Y. S. Hung, and H. Shu, "Mixed $H_{2} / H_{\infty}$ filtering for uncertain systems with regional pole assignment," IEEE Transactions on Aerospace and Electronic Systems, vol. 41, no. 2, pp. 438-448, 2005.

[61] Z. Wang, F. Yang, D. W. C. Ho, and X. Liu, "Robust varianceconstrained $H_{\infty}$ control for stochastic systems with multiplicative noises," Journal of Mathematical Analysis and Applications, vol. 328, no. 1, pp. 487-502, 2007.

[62] K.-Y. Chang and W.-J. Wang, "Robust covariance control for perturbed stochastic multivariable system via variable structure control," Systems \& Control Letters, vol. 37, no. 5, pp. 323-328, 1999.

[63] K.-Y. Chang and W.-J. Wang, " $H_{\infty}$ norm constraint and variance control for stochastic uncertain large-scale systems via the sliding mode concept," IEEE Transactions on Circuits and Systems I, vol. 46, no. 10, pp. 1275-1280, 1999.

[64] W.-J. Wang and K.-Y. Chang, "Variable structure-based covariance assignment for stochastic multivariable model reference systems," Automatica, vol. 36, no. 1, pp. 141-146, 2000. 
[65] A. Subramanian and A. H. Sayed, "Multi-objective filter design for uncertain stochastic time-delay systems," IEEE Transactions on Automatic Control, vol. 49, no. 1, pp. 149-154, 2004.

[66] L. Ma, Z. Wang, Y. Bo, and Z. Guo, "Robust fault-tolerant control for a class of nonlinear stochastic systems with variance constraints," Journal of Dynamic Systems, Measurement and Control, vol. 132, no. 4, Article ID 044501, 6 pages, 2010.

[67] L. Ma, Z. Wang, J. Hu, Y. Bo, and Z. Guo, "Robust varianceconstrained filtering for a class of nonlinear stochastic systems with missing measurements," Signal Processing, vol. 90, no. 6, pp. 2060-2071, 2010.

[68] L. Ma, Z. Wang, Y. Bo, and Z. Guo, "Finite-horizon $H_{2} / H_{\infty}$ control for a class of nonlinear Markovian jump systems with probabilistic sensor failures," International Journal of Control, vol. 84, no. 11, pp. 1847-1857, 2011.

[69] L. Guo, F. Yang, and J. Fang, "Multi-objective filtering for nonlinear time-delay systems with nonzero initial conditions based on convex optimization," Circuits, Systems, \& Signal Processing, vol. 25, no. 5, pp. 591-607, 2006.

[70] P. Shi, M. S. Mahmoud, J. Yi, and A. Ismail, "Worst case control of uncertain jumping systems with multi-state and input delay information," Information Sciences, vol. 176, no. 2, pp. 186-200, 2006.

[71] E. Tian and C. Peng, "Delay-dependent stability analysis and synthesis of uncertain T-S fuzzy systems with time-varying delay," Fuzzy Sets \& Systems, vol. 157, no. 4, pp. 544-559, 2006.

[72] D. Yue and J. Lam, "Non-fragile guaranteed cost control for uncertain descriptor systems with time-varying state and input delays," Optimal Control Applications \& Methods, vol. 26, no. 2, pp. 85-105, 2005.

[73] S. Elmadssia, K. Saadaoui, and M. Benrejeb, "New delaydependent stability conditions for linear systems with delay," Systems Science and Control Engineering: An Open Access Journal, vol. 1, no. 1, pp. 2-11, 2013.

[74] M. Darouach and M. Chadli, "Admissibility and control of switched discrete-time singular systems," Systems Science and Control Engineering: An Open Access Journal, vol. 1, no. 1, pp. 43-51, 2013.

[75] G. Zames, "Feedback and optimal sensitivity: model reference transformations, multiplicative seminorms, and approximate inverses," IEEE Transactions on Automatic Control, vol. 26, no. 2, pp. 301-320, 1981.

[76] J. C. Doyle, K. Glover, P. P. Khargonekar, and B. A. Francis, "State-space solutions to standard $H_{2}$ and $H_{\infty}$ control problems," IEEE Transactions on Automatic Control, vol. 34, no. 8, pp. 831-847, 1989.

[77] A. Isidori and W. Kang, " $H_{\infty}$ control via measurement feedback for general nonlinear systems," IEEE Transactions on Automatic Control, vol. 40, no. 3, pp. 466-472, 1995.

[78] A. J. van der Schaft, " $L_{2}$-gain analysis of nonlinear systems and nonlinear state feedback $H_{\infty}$ control," IEEE Transactions on Automatic Control, vol. 37, no. 6, pp. 770-784, 1992.

[79] Z. Wang, D. Ding, H. Dong, and H. Shu, " $H_{\infty}$ consensus control for multi-agent systems with missing measurements: the finitehorizon case," Systems \& Control Letters, vol. 62, no. 10, pp. 827836, 2013.

[80] L. Ma, Z. Wang, Y. Bo, and Z. Guo, "Robust $H_{\infty}$ control of time-varying systems with stochastic non-linearities: the finite-horizon case," Proceedings of the Institution of Mechanical Engineers I, vol. 224, no. 5, pp. 575-585, 2010.
[81] L. Ma, Z. Wang, Y. Bo, and Z. Guo, "Robust $H_{\infty}$ sliding mode control for nonlinear stochastic systems with multiple data packet losses," International Journal of Robust and Nonlinear Control, vol. 22, no. 5, pp. 473-491, 2012.

[82] D. Ding, Z. Wang, H. Dong, and H. Shu, "Distributed $H_{\infty}$ state estimation with stochastic parameters and nonlinearities through sensor networks: the finite-horizon case," Automatica, vol. 48, no. 8, pp. 1575-1585, 2012.

[83] J. Hu, Z. Wang, Y. Niu, and L. K. Stergioulas, " $H_{\infty}$ sliding mode observer design for a class of nonlinear discrete time-delay systems: a delay-fractioning approach," International Journal of Robust and Nonlinear Control, vol. 22, no. 16, pp. 1806-1826, 2012.

[84] Z. Wang, H. Dong, B. Shen, and H. Gao, "Finite-horizon $H_{\infty}$ filtering with missing measurements and quantization effects," IEEE Transactions on Automatic Control, vol. 58, no. 7, pp. 17071718, 2013.

[85] H. Dong, Z. Wang, and H. Gao, "Distributed $H_{\infty}$ filtering for a class of Markovian jump nonlinear time-delay systems over lossy sensor networks," IEEE Transactions on Industrial Electronics, vol. 60, no. 10, pp. 4665-4672, 2013.

[86] Z. Wang, D. W. C. Ho, Y. Liu, and X. Liu, "Robust $H_{\infty}$ control for a class of nonlinear discrete time-delay stochastic systems with missing measurements," Automatica, vol. 45, no. 3, pp. 684-691, 2009.

[87] Z. Shu, J. Lam, and J. Xiong, "Non-fragile exponential stability assignment of discrete-time linear systems with missing data in actuators," IEEE Transactions on Automatic Control, vol. 54, no. 3, pp. 625-630, 2009.

[88] G. Wei, Z. Wang, and H. Shu, "Robust filtering with stochastic nonlinearities and multiple missing measurements," Automatica, vol. 45, no. 3, pp. 836-841, 2009.

[89] F. Yang and Y. Li, "Set-membership filtering for systems with sensor saturation," Automatica, vol. 45, no. 8, pp. 1896-1902, 2009.

[90] G.-H. Yang and D. Ye, "Adaptive reliable $H_{\infty}$ filtering against sensor failures," IEEE Transactions on Signal Processing, vol. 55, no. 7, part 1, pp. 3161-3171, 2007.

[91] M. Fu and C. E. de Souza, "State estimation for linear discretetime systems using quantized measurements," Automatica, vol. 45, no. 12, pp. 2937-2945, 2009.

[92] N. Xiao, L. Xie, and M. Fu, "Stabilization of Markov jump linear systems using quantized state feedback," Automatica, vol. 46, no. 10, pp. 1696-1702, 2010.

[93] X.-Z. Dong, "Robust strictly dissipative control for discrete singular systems," IET Control Theory \& Applications, vol. 1, no. 4, pp. 1060-1067, 2007.

[94] D. Hill and P. Moylan, "The stability of nonlinear dissipative systems," IEEE Transactions on Automatic Control, vol. 21, no. 5, pp. 708-711, 1976.

[95] Z. Li, J. Wang, and H. Shao, "Delay-dependent dissipative control for linear time-delay systems," Journal of the Franklin Institute, vol. 339, no. 6-7, pp. 529-542, 2002.

[96] Z. Tan, Y. C. Soh, and L. Xie, "Dissipative control for linear discrete-time systems," Automatica, vol. 35, no. 9, pp. 1557-1564, 1999.

[97] J. C. Willems, "Dissipative dynamical systems, part 1: general theory; part 2: linear systems with quadratic supply rate," Archive for Rational Mechanics and Analysis, vol. 45, no. 5, pp. 321-393, 1972. 
[98] S. Xie, L. Xie, and C. E. de Souza, "Robust dissipative control for linear systems with dissipative uncertainty," International Journal of Control, vol. 70, no. 2, pp. 169-191, 1998.

[99] A. Mehrsai, H. R. Karimi, and K.-D. Thoben, "Integration of supply networks for customization with modularity in cloud and make-to-upgrade strategy," Systems Science and Control Engineering: An Open Access Journal, vol. 1, no. 1, pp. 28-42, 2013.

[100] Z. Wang, J. Lam, L. Ma, Y. Bo, and Z. Guo, "Varianceconstrained dissipative observer-based control for a class of nonlinear stochastic systems with degraded measurements," Journal of Mathematical Analysis and Applications, vol. 377, no. 2, pp. 645-658, 2011.

[101] H. Dong, Z. Wang, and H. Gao, " $H_{\infty}$ fuzzy control for systems with repeated scalar nonlinearities and random packet losses," IEEE Transactions on Fuzzy Systems, vol. 17, no. 2, pp. 440-450, 2009.

[102] J. Liang, Z. Wang, and X. Liu, "On passivity and passification of stochastic fuzzy systems with delays: the discrete-time case," IEEE Transactions on Systems, Man, and Cybernetics B, vol. 40, no. 3, pp. 964-969, 2010.

[103] H. Dong, Z. Wang, D. W. C. Ho, and H. Gao, "Robust $H_{\infty}$ fuzzy output-feedback control with multiple probabilistic delays and multiple missing measurements," IEEE Transactions on Fuzzy Systems, vol. 18, no. 4, pp. 712-725, 2010.

[104] H. Dong, Z. Wang, J. Lam, and H. Gao, "Fuzzy-model-based robust fault detection with stochastic mixed time delays and successive packet dropouts," IEEE Transactions on Systems, Man, and Cybernetics B, vol. 42, no. 2, pp. 365-376, 2012.

[105] B.-K. Lee, C.-H. Chiu, and B.-S. Chen, "Adaptive minimum variance control for stochastic fuzzy T-S ARMAX model," in Proceedings of the 7th International Conference on Machine Learning and Cybernetics (ICMLC'08), pp. 3811-3816, Kunming, China, July 2008.

[106] W.-J. Chang and S.-M. Wu, "State variance constrained fuzzy controller design for nonlinear TORA systems with minimizing control input energy," in Proceedings of the IEEE International Conference on Robotics and Automation, pp. 2616-2621, September 2003.

[107] W.-J. Chang and S.-M. Wu, "Continuous fuzzy controller design subject to minimizing control input energy with output variance constraints," European Journal of Control, vol. 11, no. 3 , pp. 269-277, 2005.

[108] W. J. Chang and B. J. Huang, "Passive fuzzy controller design with variance constraint for nonlinear synchronous generator systems," in Proceedings of the IEEE 10th International Conference on Power Electronics and Drive Systems, pp. 1251-1256, 2013.

[109] V. I. Utkin, "Variable structure systems with sliding modes," IEEE Transactions on Automatic Control, vol. 22, no. 2, pp. 212222, 1977.

[110] Y. Niu, D. W. C. Ho, and J. Lam, "Robust integral sliding mode control for uncertain stochastic systems with time-varying delay," Automatica, vol. 41, no. 5, pp. 873-880, 2005.

[111] Y. Niu and D. W. C. Ho, "Robust observer design for Itô stochastic time-delay systems via sliding mode control," Systems \& Control Letters, vol. 55, no. 10, pp. 781-793, 2006.

[112] K. Abidi, J.-X. Xu, and X. Yu, "On the discrete-time integral sliding-mode control," IEEE Transactions on Automatic Control, vol. 52, no. 4, pp. 709-715, 2007.

[113] C. Bonivento, M. Sandri, and R. Zanasi, "Discrete variablestructure integral controllers," Automatica, vol. 34, no. 3, pp. 355-361, 1998.
[114] C. Y. Chan, "Discrete adaptive sliding-mode control of a class of stochastic systems," Automatica, vol. 35, no. 8, pp. 1491-1498, 1999.

[115] X. Chen and T. Fukuda, "Robust adaptive quasi-sliding mode controller for discrete-time systems," Systems \& Control Letters, vol. 35, no. 3, pp. 165-173, 1998.

[116] C.-C. Cheng, M.-H. Lin, and J.-M. Hsiao, "Sliding mode controllers design for linear discrete-time systems with matching perturbations," Automatica, vol. 36, no. 8, pp. 1205-1211, 2000.

[117] C.-L. Hwang, "Robust discrete variable structure control with finite-time approach to switching surface," Automatica, vol. 38, no. 1, pp. 167-175, 2002.

[118] N. O. Lai, C. Edwards, and S. K. Spurgeon, "Discrete output feedback sliding-mode control with integral action," International Journal of Robust and Nonlinear Control, vol. 16, no. 1, pp. 21-43, 2006.

[119] N. O. Lai, C. Edwards, and S. K. Spurgeon, "On output tracking using dynamic output feedback discrete-time sliding-mode controllers," IEEE Transactions on Automatic Control, vol. 52, no. 10, pp. 1975-1981, 2007.

[120] W. Gao, Y. Wang, and A. Homaifa, "Discrete-time variable structure control systems," IEEE Transactions on Industrial Electronics, vol. 42, no. 2, pp. 117-122, 1995.

[121] Y. Xia, G. P. Liu, P. Shi, J. Chen, D. Rees, and J. Liang, "Sliding mode control of uncertain linear discrete time systems with input delay," IET Control Theory \& Applications, vol. 1, no. 4, pp. 1169-1175, 2007.

[122] M. Yan and Y. Shi, "Robust discrete-time sliding mode control for uncertain systems with time-varying state delay," IET Control Theory \& Applications, vol. 2, no. 8, pp. 662-674, 2008.

[123] D. S. Bernstein and W. M. Haddad, "LQG control with an $H_{\infty}$ performance bound: a Riccati equation approach," IEEE Transactions on Automatic Control, vol. 34, no. 3, pp. 293-305, 1989.

[124] D. J. N. Limebeer, B. D. O. Anderson, and B. Hendel, "A Nash game approach to mixed $H_{2} / H_{\infty}$ control," IEEE Transactions on Automatic Control, vol. 39, no. 1, pp. 69-82, 1994.

[125] P. P. Khargonekar and M. A. Rotea, "Mixed $H_{2} / H_{\infty}$ control: a convex optimization approach," IEEE Transactions on Automatic Control, vol. 36, no. 7, pp. 824-837, 1991.

[126] L. Zhang, B. Huang, and J. Lam, "LMI synthesis of $H_{2}$ and mixed $\mathrm{H}_{2} / \mathrm{H}_{\mathrm{\infty}}$ controllers for singular systems," IEEE Transactions on Circuits and Systems II, vol. 50, no. 9, 2003.

[127] C. Scherer, P. Gahinet, and M. Chilali, "Multi-objective outputfeedback control via LMI optimization," IEEE Transactions on Automatic Control, vol. 42, no. 7, pp. 896-911, 1997.

[128] M.-J. Khosrowjerdi, R. Nikoukhah, and N. Safari-Shad, "Fault detection in a mixed $\mathrm{H}_{2} / \mathrm{H}_{\infty}$ setting," in Proceedings of the 42nd IEEE Conference on Decision and Control, pp. 1461-1466, December 2003.

[129] C.-D. Yang and Y.-P. Sun, "Mixed $H_{2}$ and mixed $H_{2} / H_{\infty}$ statefeedback design for microsatellite attitude control," Control Engineering Practice, vol. 10, no. 9, pp. 951-970, 2002.

[130] X. Chen and K. Zhou, "Multi-objective filtering design," in Proceedings of the IEEE Canadian Conference on Electrical and Computer Engineering, pp. 708-713, May 1999.

[131] Y. Theodor and U. Shaked, "A dynamic game approach to mixed $\mathrm{H}_{2}$ and mixed $\mathrm{H}_{2} / \mathrm{H}_{\infty}$ estimation," International Journal of Robust and Nonlinear Control, vol. 6, no. 4, pp. 331-345, 1996.

[132] Z. Wang and B. Huang, "Robust $H_{2} / H_{\infty}$ filtering for linear systems with error variance constraints," IEEE Transactions on Signal Processing, vol. 48, no. 8, pp. 2463-2467, 2000. 
[133] W. Lin, "Mixed $\mathrm{H}_{2} / \mathrm{H}_{\infty}$ control of nonlinear systems," in Proceedings of the 34th IEEE Conference on Decision and Control, pp. 333-338, December 1995.

[134] B.-S. Chen, C.-S. Tseng, and H.-J. Uang, "Mixed $H_{2} / H_{\infty}$ fuzzy output feedback control design for nonlinear dynamic systems: an LMI approach," IEEE Transactions on Fuzzy Systems, vol. 8, no. 3, pp. 249-265, 2000.

[135] F. Yang, Z. Wang, and D. W. C. Ho, "Robust mixed $H_{2} / H_{\infty}$ control for a class of nonlinear stochastic systems," IEE Proceedings on Control Theory and Applications, vol. 153, no. 2, pp. 175-184, 2006. 


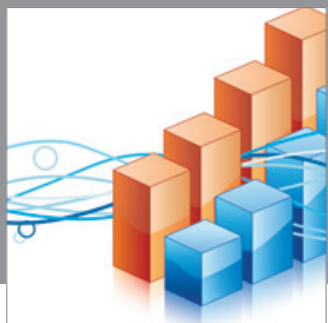

Advances in

Operations Research

mansans

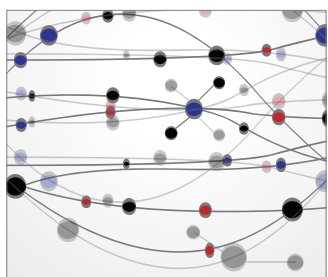

The Scientific World Journal
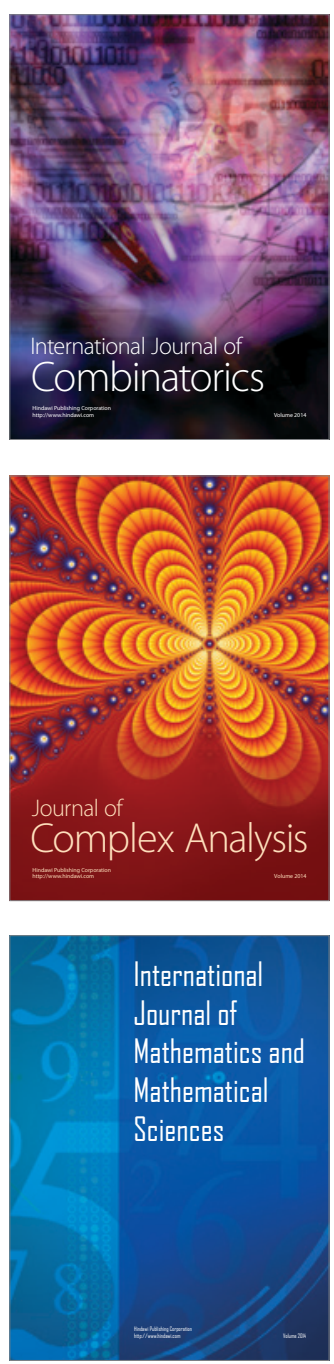
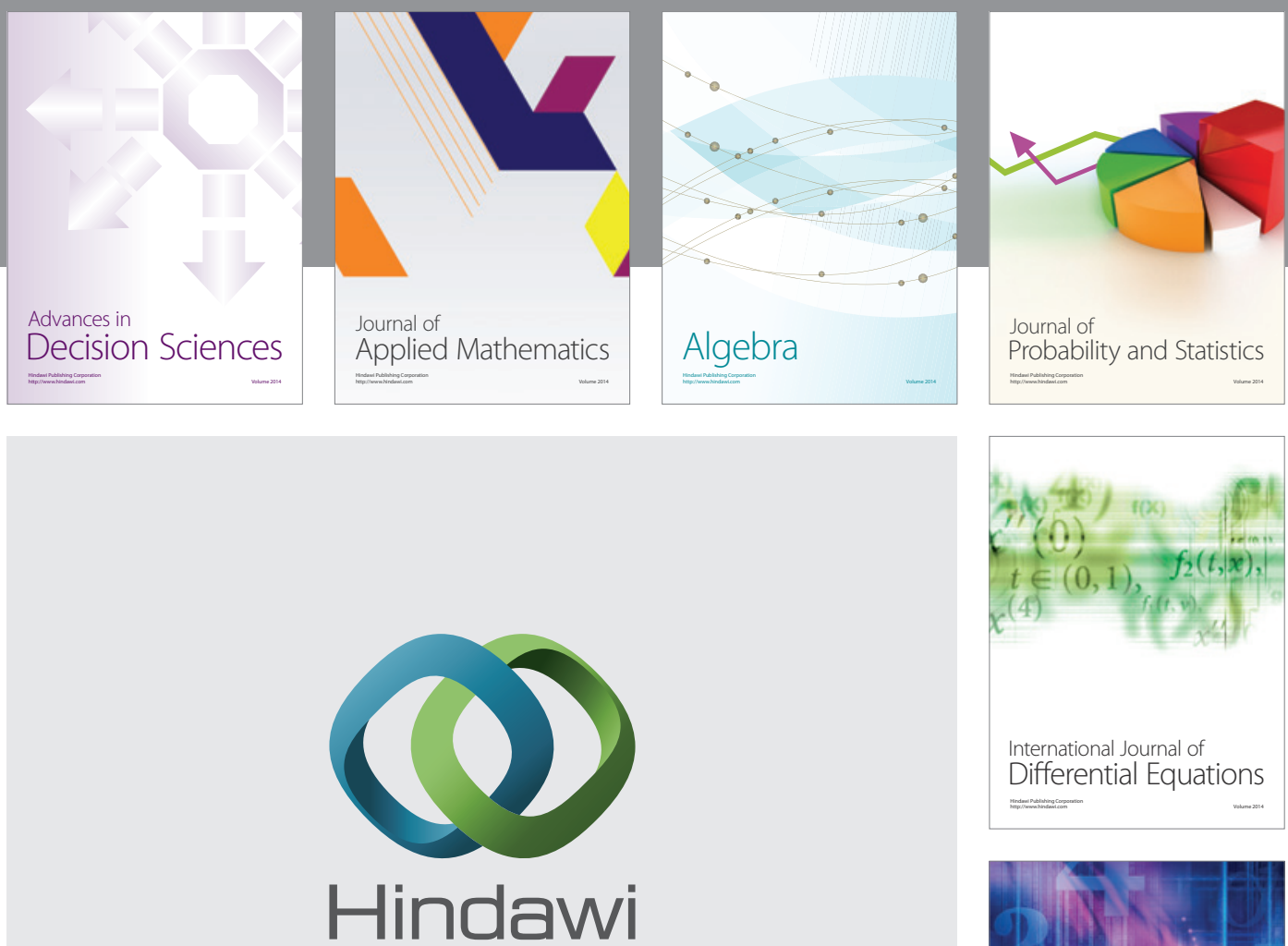

Submit your manuscripts at http://www.hindawi.com
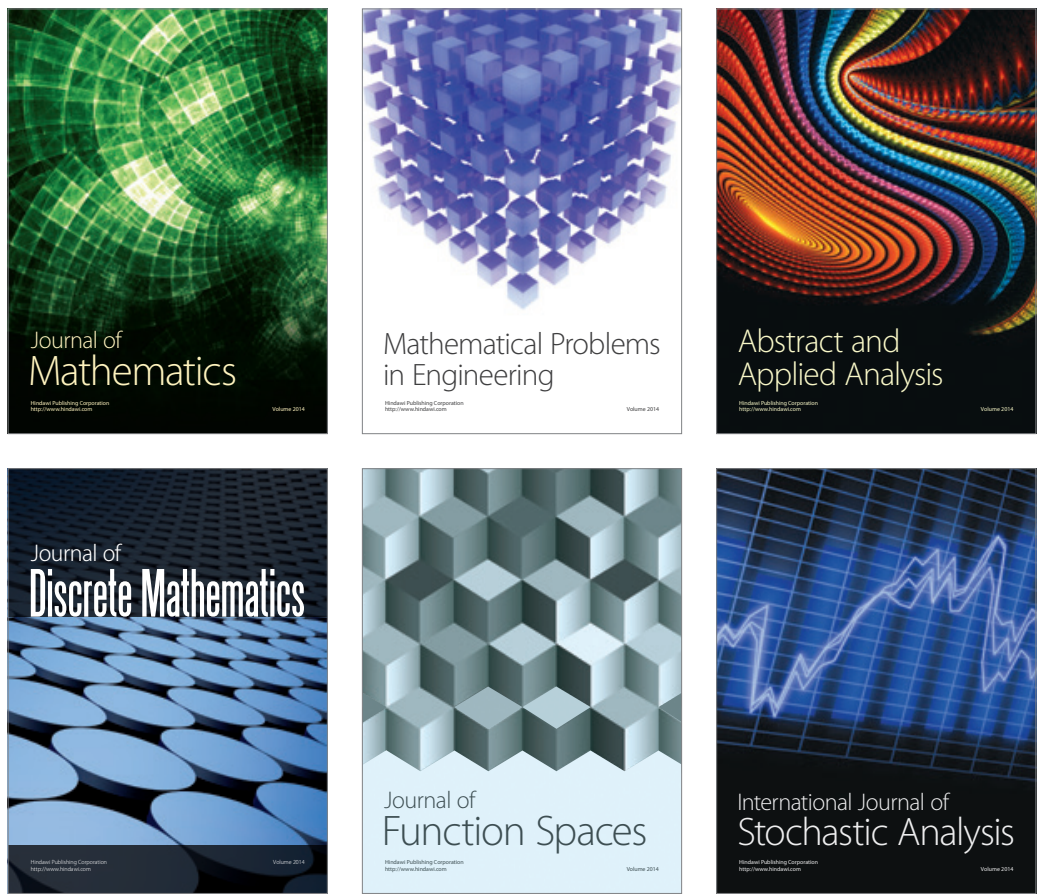

Journal of

Function Spaces

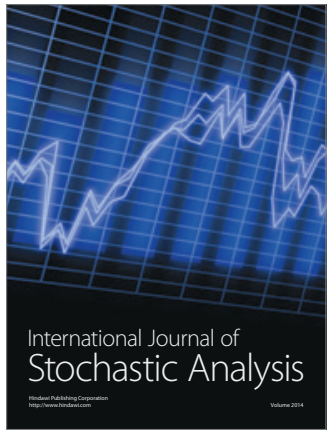

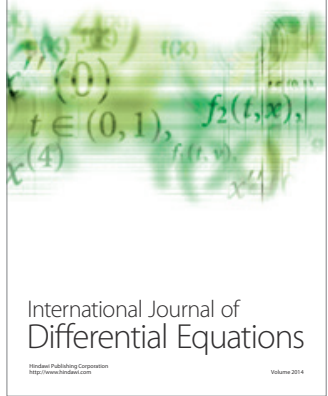
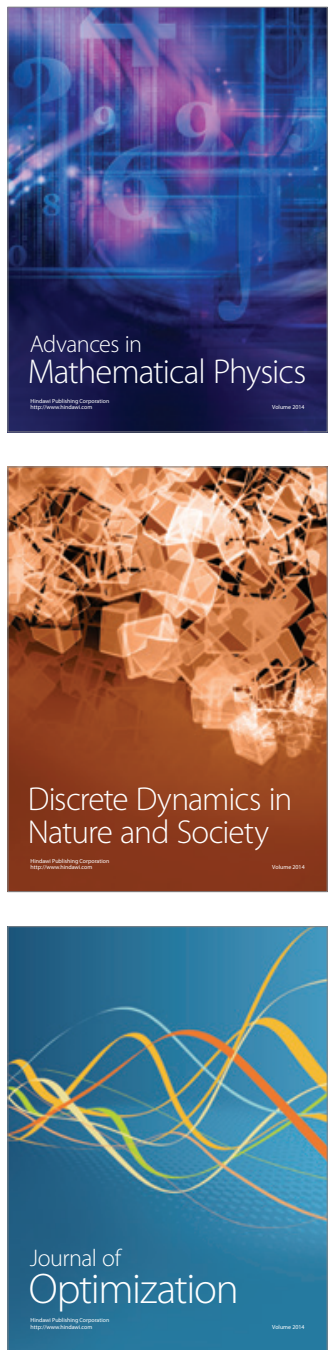